\title{
MULTIPLE BIFURCATIONS OF A CYLINDRICAL DYNAMICAL SYSTEM*
}

\author{
Ning Han, Qinguie CaO \\ Centre for Nonlinear Dynamics Research, \\ Harbin Institute of Technology, \\ School of Astronautics, Harbin 150001 China, \\ e-mails: han_ning@outlook.com, q.j.cao@hit.edu.cn
}

[Received 05 June 2015, Accepted 29 February 2016]

\begin{abstract}
This paper focuses on multiple bifurcations of a cylindrical dynamical system, which is evolved from a rotating pendulum with SD oscillator. The rotating pendulum system exhibits the coupling dynamics property of the bistable state and conventional pendulum with the homoclinic orbits of the first and second type. A double Andronov-Hopf bifurcation, two saddle-node bifurcations of periodic orbits and a pair of homoclinic bifurcations are detected by using analytical analysis and numerical calculation. It is found that the homoclinic orbits of the second type can bifurcate into a pair of rotational limit cycles, coexisting with the oscillating limit cycle. Additionally, the results obtained herein, are helpful to explore different types of limit cycles and the complex dynamic bifurcation of cylindrical dynamical system.

KEY WORDS: Cylindrical dynamical system, rotating pendulum with SD oscillator, homoclinic bifurcation, saddle-node bifurcations of periodic orbits, limit cycle.
\end{abstract}

\section{Introduction}

The cylindrical dynamical system [1, 2, 3] has received a resurgence of attention in recent years, owing to the wide range of application in civil, military and industrial applications, such as horologium, robot [4], pendulum

\footnotetext{
${ }^{*}$ Corresponding author e-mail: q.j.cao@hit.edu.cn

The authors would like to acknowledge the financial support from the Natural Science Foundation of China (Grant Nos 11372082 and 11572096) and the National Basic Research Program of China (Grant No. 2015CB057405).
} 
absorber [5], gas turbine rotors [6], as well. It is well known, that the conventional pendulum is a typically cylindrical dynamical system, which is among the most widely investigated motions in physics and many nonlinear phenomena in the real world. Galileo found the law of isochronism through classical experiments in the 16th century, which marked the beginning of the scientific research of cylindrical pendulum. In the 17th century, Huygens discovered the periodicity of large swings, deviating from the law isochronism, which is one of the earliest records of cylindrical pendulum. In modern time, many kinds of pendulum-like system or systems containing the pendulum have been proposed and investigated, which greatly enrich the research content of cylindrical dynamical system. For example, the chaotic dynamics and subharmonic bifurcations in a pendulum-like system were studied in [7]. The approximate analytical solutions for oscillatory and rotational motion of a parametric pendulum were discussed in [8]. The bifurcation in an inverted pendulum with the high frequency excitation was proved by using analytical and experimental investigations in [9]. Subharmonic and homoclinic bifurcations in a parametrically forced pendulum was studied in [10]. Recently, a rotating pendulum linked by an oblique spring with fixed end has been proposed and investigated in [11]. This system is a cylindrical dynamical system with irrational characters, whose free motion is similar to the conventional pendulum coupled with SD oscillator $[12,13,14]$ of the homoclinic orbits of the first and second type. The authors introduce a cylindrical approximate system, for which the analytical solutions can be obtained successfully to reflect the nature of the rotating pendulum system, without barrier of the irrationalities [15]. Then, the chaotic boundary of discontinuous case with a pair of heteroclinic-like orbits [16] are obtained by using semi-analytical method. Throughout the hot research topics of cylindrical dynamical system, very few attempts have been made in reporting the oscillating and rotational limit cycles of the autonomous system, which is of fundamental importance to reveal the essential phenomenology of cylindrical dynamical system from the view of nonlinear science and may be helpful to explore directly the bifurcation of the cylindrical dynamical system avoiding Taylor's expansion.

The aim of this paper is to start exploring multiple bifurcations of a cylindrical dynamical system, which is evolved from a rotating pendulum linked by a vertical spring with sliding end under a nonlinear perturbation. The other motivation of this work is to provide a typical example to reveal the cylindrical phenomena by means of the results obtained herein. Particularly, it is found that the homoclinic orbits of the second type can bifurcate into a pair of rotational limit cycles coexisting with the oscillating limit cycle. 
This paper is organized as follows: In Section 2, the mathematical model for the cylindrical pendulum system with a nonlinear perturbation is developed. In the following section, Section 3, the unperturbed dynamics of the pendulum system is directly analyzed by using nonlinear dynamical technique. While in Section 4, a double Andronov-Hopf bifurcation, two saddle-node bifurcations of periodic orbits and a pair of homoclinic bifurcations are investigated by using analytical analysis and numerical calculation. Finally this paper is ended with conclusions.

\section{Cylindrical dynamical system}

Consider the simple model which comprises a rotating pendulum linked by a vertical spring with sliding end, as shown in Fig. 1. It is assumed to oscillate or rotate in the vertical plane. The equation of motion is given by:

$$
m L \ddot{x}-m g \sin x-k \sin x(h-l+L \cos x)=0,
$$

where the dot denotes derivative with respect to $t, x$ is the angular displacement, $m$ and $L$ are the lump mass and pendulum length, $k$ and $l$ are the stiffness and relax length of the spring, $h$ is the height between A and B. Again, system (1) can be written as:

$$
\ddot{x}-\sin x(\alpha+\beta \cos x)=0, \quad \alpha=\frac{1}{L}\left(g+\frac{k}{m}(h-l)\right), \quad \beta=\frac{k}{m} .
$$

From the mathematical perspective, parameters $\alpha$ and $\beta$ can be regarded as mutually independent. From a physical standpoint, parameter $\alpha$ defines the

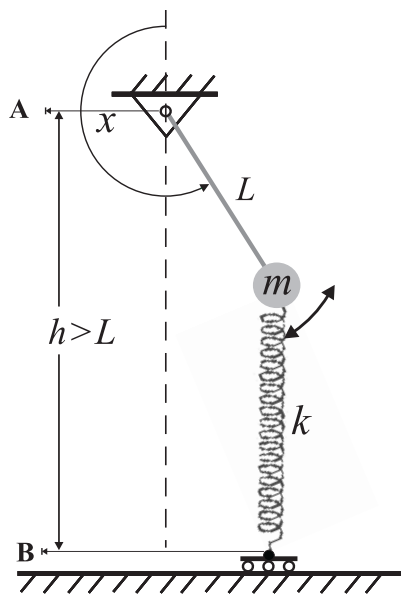

Fig. 1. The model of a rotating pendulum linked by a vertical spring with sliding end 
geometry of the physical model and $\beta$ mainly reflects the system stiffness. Assuming that system (1) is perturbed by a nonlinear perturbation $(-c+$ $\left.d \cos ^{2} x\right) \dot{x}$, the forced dissipative system can be derived as a two dimensional one:

(3) $\dot{x}=y, \quad \dot{y}=\sin x(\alpha+\beta \cos x)-\left(-\xi+\delta \cos ^{2} x\right) y, \quad \xi=\frac{c}{m L}, \quad \delta=\frac{d}{m L}$,

where $\xi$ and $\delta$ are positive small parameters. Letting $X=\cos x, Y=\sin x, Z=$ $y$, system (3) can be re-written in the following cylindrical form:

(4) $\dot{X}=-Y Z, \quad \dot{Y}=X Z, \quad \dot{Z}=Y(\alpha+\beta X)-\left(-\xi+\delta X^{2}\right) Z, \quad X^{2}+Y^{2}=1$.

It is noticing that the perturbation term $\left(-\xi+\delta X^{2}\right) Z$ in system (4) is similar to the Vanderpol oscillator $[17,18]$.

\section{Unperturbed dynamics}

In this section, the unperturbed dynamics of the rotating pendulum system including the equilibria, Hamiltonian function, homoclinic orbits and phase portraits are discussed by using nonlinear dynamical technique, respectively.

The unperturbed system (2) can be written as a two dimensional one:

$$
\dot{x}=y, \quad \dot{y}=\sin x(\alpha+\beta \cos x), \quad|\alpha|<\beta,
$$

of which the equilibria can be easily obtained by letting $\sin x(\alpha+\beta \cos x)=0$ : (6)

$$
\left(x_{1}, y_{1}\right)=(0,0), \quad\left(x_{2,3}, y_{2,3}\right)=( \pm \pi, 0), \quad\left(x_{4,5}, y_{4,5}\right)=( \pm \arccos (-\alpha / \beta), 0),
$$

where $\left(x_{4,5}, y_{4,5}\right)$ exist only for $|-\alpha / \beta|<1$. It is worth reiterating here that system (2) bears significant similarities to conventional pendulum system for $|\alpha| \geq \beta$. The Hamiltonian function of system (5) can be obtained in the following form:

$$
H(x, y)=\frac{y^{2}}{2}+\alpha \cos x-\frac{\beta}{2} \sin ^{2} x-\alpha .
$$

and the three-dimensional surface of Hamiltonian function in $\{\mathrm{x}, \mathrm{y}, \mathrm{H}(\mathrm{x}, \mathrm{y})\}$ space is plotted for $\alpha=-1, \beta=2$, as shown in Fig. 2(a). With the help of Hamiltonian function (7), the trajectories of system (5) can be classified by means of $H(x, y)=h$. In order to understand the trajectories of phase portraits as the parameter $h$ vary, we define the trajectories as $\Gamma_{h}=\{(x, y) \mid H(x, y)=h\}$. 
(a)

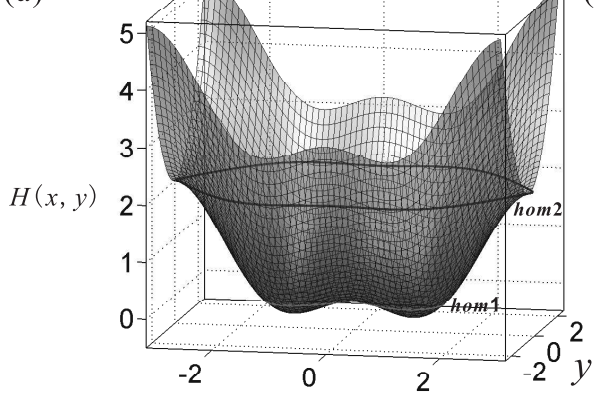

$x$

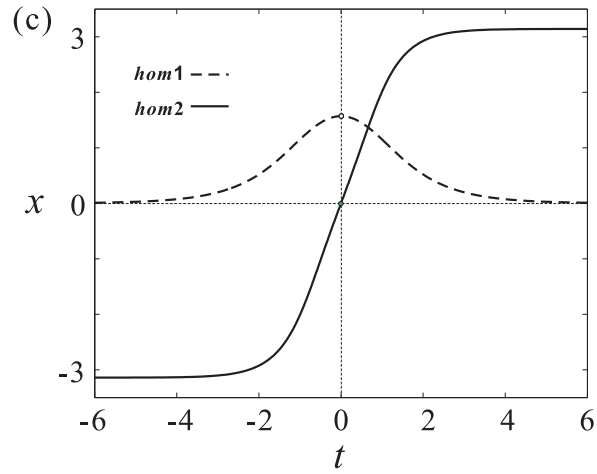

(b)

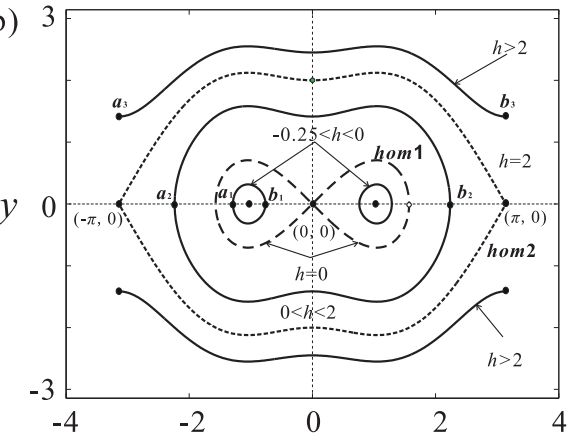

(d)

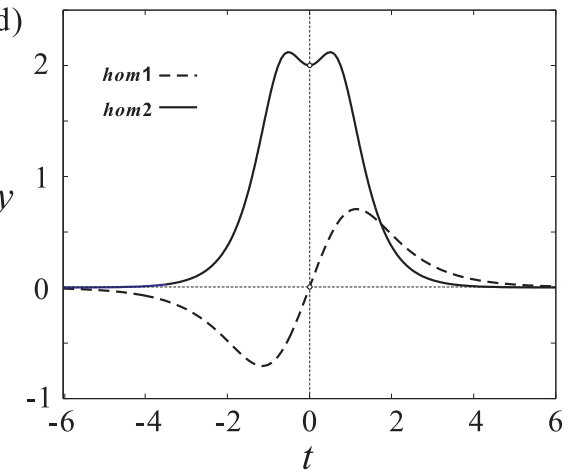

Fig. 2. When $\alpha=-1$ and $\beta=2$ : (a) the surface diagram of Hamiltonian function for the unperturbed system (5), (b) phase portrait for system $(5): \Gamma_{h}=\{(x, y) \mid H(x, y)=$ $h\}$, (c) the analytical solution $x(\mathrm{t})$ of the homoclinic orbits of the first and second type and $(\mathrm{d})$ corresponding the solution of velocity $y(\mathrm{t})$

$\Gamma_{h}$ represents a pair of closed orbits for $h \in\left(-(\alpha+\beta)^{2} / 2 \beta, 0\right)$; When $h=$ $-(\alpha+\beta)^{2} / 2 \beta, \Gamma_{h}$ shrinks into the pair of equilibria $\left(x_{4,5}, y_{4,5}\right)$. On the other side, $\Gamma_{h}$ extends into a double homoclinic orbits connecting the equilibrium $\left(x_{1}, y_{1}\right)$ for $h=0$. While $h \in(0,-2 \alpha), \Gamma_{h}$ gives the large closed orbit encircling the equilibria $\left(x_{1}, y_{1}\right),\left(x_{4,5}, y_{4,5}\right)$ and $\Gamma_{h}$ extends into a double connected homoclinic orbits of second type connecting the equilibria $\left(x_{2}, y_{3}\right)$ and $\left(x_{3}, y_{3}\right)$, when $h=-2 \alpha$. It is worth noticing, that this large closed orbit for $\Gamma_{h \in(0,-2 \alpha)}$ does shrink itself into the double homoclinic orbits of first type as $h \rightarrow 0^{+}$, on the contrary, it does extend itself into the double homoclinic orbits of second type as $h \rightarrow(-2 \alpha)^{-}$. While $h>-2 \alpha$, the double homoclinic orbits of second type $\Gamma_{h=-2 \alpha}$ divide into a pair of wavelike trajectories near the homoclinic orbits of second type. Take $\alpha=-1$ and $\beta=2$ for example, the detailed 
classification for the trajectories of phase portraits are shown in Fig. 2(b).

The double connected homoclinic orbits of first type denoted by hom 1 , for $h=0$, can be written in the parametric form as:

(8)

$$
\begin{aligned}
& \left(x_{ \pm}^{\text {hom } 1}(t), y_{ \pm}^{\text {hom } 1}(t)\right)= \\
& \left( \pm 2 \cot ^{-1}\left(\sqrt{\frac{-\alpha}{\alpha+\beta}} \cosh (\sqrt{\alpha+\beta} t)\right), \mp \frac{2 \sqrt{-\alpha} \sinh (\sqrt{\alpha+\beta} t)}{1+\frac{-\alpha}{\alpha+\beta} \cosh ^{2}(\sqrt{\alpha+\beta} t)}\right)
\end{aligned}
$$

and is plotted for $\alpha=-1$ and $\beta=2$ in Fig. 2(c) and (d) with dashed line. Similarly, the double connected homoclinic orbits of second type marked by hom 2 , for $h=-2 \alpha$, can be written in the parametric form as:

(9)

$$
\begin{aligned}
& \left(x_{ \pm}^{\text {hom } 2}(t), y_{ \pm}^{\text {hom } 2}(t)\right)= \\
& \left( \pm 2 \tan ^{-1}\left(\sqrt{\frac{-\alpha}{\beta-\alpha}} \sinh (\sqrt{\beta-\alpha} t)\right), \mp \frac{2 \sqrt{-\alpha} \cosh (\sqrt{\beta-\alpha} t)}{1+\frac{-\alpha}{\beta-\alpha} \sinh ^{2}(\sqrt{\beta-\alpha} t)}\right)
\end{aligned}
$$

and is displayed for $\alpha=-1$ and $\beta=2$ in Fig. 2(c) and (d) with solid line.

For $\xi=\delta=0$, the cylindrical form (4) is given by

$$
\dot{X}=-Y Z, \dot{Y}=X Z, \dot{Z}=Y(\alpha+\beta X), X^{2}+Y^{2}=1 .
$$

The equilibria of system (10) can be easily derived by letting $\dot{X}=0, \dot{Y}=$ $0, \dot{Z}=0$, and written as:

$$
\left(X_{1,2}, Y_{1,2}, Z_{1,2}\right)=( \pm 1,0,0),\left(X_{3,4}, Y_{3,4}, Z_{3,4}\right)=\left(-\frac{\alpha}{\beta}, \pm \frac{\sqrt{\beta^{2}-\alpha^{2}}}{\beta}, 0\right)
$$

Multiplying both sides of the second and third equation by the first equation in system (10) leads to:

$$
X \dot{X}=-Y \dot{Y}, \quad-Z \dot{Z}=(\alpha+\beta X) \dot{X} .
$$

Integrating both sides of system (12), the Hamiltonian function of system (10) can be derived:

$$
H(X, Y, Z)=\frac{Z^{2}}{2}+\alpha X+\frac{\beta}{2} X^{2}, \quad X^{2}+Y^{2}=1 .
$$


The phase portraits plotted via Hamiltonian function (13) $H(X, Y, Z)=h$ is shown for $\alpha=-1$ and $\beta=2$ in Fig. 3(a). It is more interesting that a pair of homoclinic orbits of the first type connecting the saddle point $\left(X_{1}, Y_{1}, Z_{1}\right)$ and the second type connecting the saddle point $\left(X_{2}, Y_{2}, Z_{2}\right)$, (hom 1 and hom 2 ), are co-existed in Fig. 3(a). The homoclinic orbits of the first type connected

(a)

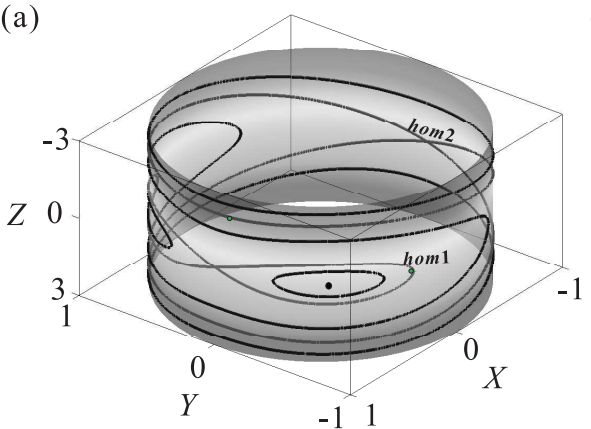

(c)

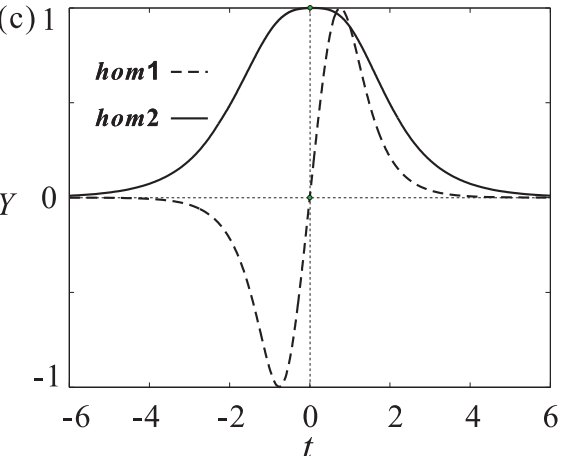

(b)

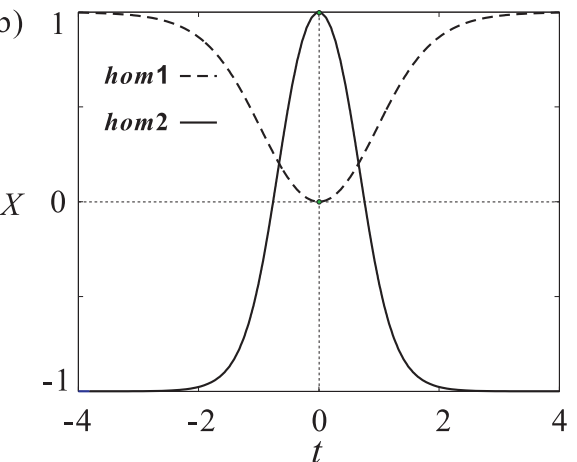

(d)

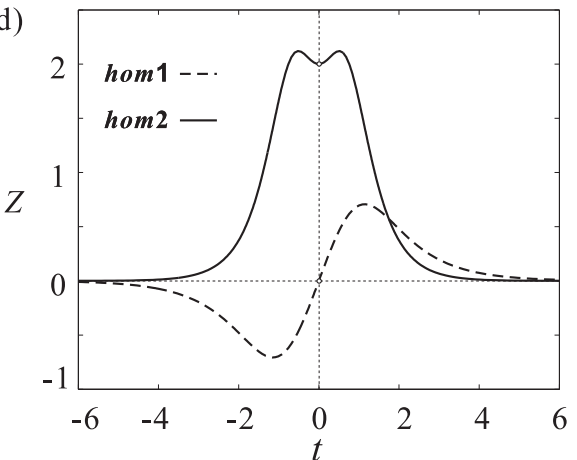

Fig. 3. When $\alpha=-1$ and $\beta=2$ : (a) cylindrical phase portraits: a pair of homoclinic orbits of the first (hom 1 ) and the second type (hom 2 ) with saddles at $( \pm 1,0,0)$ and centers at $(1 / 2, \pm \sqrt{3} / 2,0)$. (b)-(d) the solutions $\left(X_{ \pm}^{h o m 1,2}(t), Y_{ \pm}^{h o m 1,2}(t), Z_{ \pm}^{h o m 1,2}(t)\right)$ for a pair of homoclinic orbits of the first (dashed line) and the second type (solid line)

the equilibrium $\left(X_{1}, Y_{1}, Z_{1}\right)$, for $h=0$, can be written in the parametric form as:

$$
\begin{aligned}
\left(X_{ \pm}^{\text {hom } 1}(t), Y_{ \pm}^{\text {hom } 1}(t)\right. & \left., Z_{ \pm}^{\text {hom } 1}(t)\right) \\
& =\left(\cos \left(x_{ \pm}^{\text {hom } 1}(t)\right), \pm \sin \left(x_{ \pm}^{\text {hom } 1}(t)\right), y_{ \pm}^{\text {hom } 1}(t)\right) .
\end{aligned}
$$


The homoclinic orbits of the second type connected the equilibrium $\left(X_{2}, Y_{2}\right.$, $Z_{2}$ ), for $h=-2 \alpha$, can be written in the parametric form as

$$
\begin{aligned}
\left(X_{ \pm}^{\text {hom } 2}(t), Y_{ \pm}^{\text {hom } 2}(t)\right. & \left., Z_{ \pm}^{\text {hom } 2}(t)\right) \\
& =\left(\cos \left(x_{ \pm}^{\text {hom } 2}(t)\right), \pm \sin \left(x_{ \pm}^{\text {hom } 2}(t)\right), y_{ \pm}^{\text {hom } 2}(t)\right) .
\end{aligned}
$$

Based upon Eq. (14) and Eq. (15), a pair of homoclinic orbits of the first (dashed line) and the second type (solid line) are plotted in Fig. 3(b), (c) and (d).

\section{Perturbed dynamics}

In this section, a double Andronov-Hopf bifurcation [19], two saddlenode bifurcations of periodic orbits [20] and a pair of homoclinic bifurcations [21] are investigated for a given set of parameters by using analytical analysis and numerical calculation.

\subsection{Double Andronov-Hopf Bifurcation}

Andronov-Hopf bifurcation of nonlinear dynamical system has been paid close attention widely over the last decade. It is a phenomenon, which consists of a family of limit cycles bifurcating from an initially stable (or nonhyperbolic) equilibrium.

Then, we need to analyze the equilibrium points and their stability for the perturbed system (3). For $|\alpha|<\beta$, the Jacobian matrix of the unperturbed system (3) at equilibria $\left.\left(x_{i}, y_{i}\right)\right|_{i=1,2 \cdots 5}$ can be derived as

$$
J_{\left.\left(x_{i}, y_{i}\right)\right|_{1,2 \cdots 5}}=\left[\begin{array}{cc}
0 & 1 \\
\alpha \cos x_{i}+\beta \cos 2 x_{i}-\delta y_{i} \sin 2 x_{i} & \xi-\delta \cos ^{2} x_{i}
\end{array}\right] .
$$

The characteristic equation of Jacobian matrix yields:

$$
\lambda^{2}-\left(\xi-\delta \cos ^{2} x_{i}\right) \lambda-\left(\alpha \cos x_{i}+\beta \cos 2 x_{i}-\delta y_{i} \sin 2 x_{i}\right)=0 .
$$

It is clear, that equilibrium $\left(x_{1}, y_{1}\right)$ is a saddle point with the eigenvalues $\lambda_{1,2}=\left[(\xi-\delta) \pm \sqrt{(\xi-\delta)^{2}+4(\alpha+\beta)}\right] / 2$, and $\left(x_{2,3}, y_{2,3}\right)$ are saddle points with the eigenvalues $\lambda_{1,2}=\left[(\xi-\delta) \pm \sqrt{(\xi-\delta)^{2}+4(\beta-\alpha)}\right] / 2$, respectively. The Jacobian matrix for system $(3)$ at $\left(x_{4,5}, y_{4,5}\right)$ is given by:

$$
J_{\left(x_{4,5}, y_{4,5}\right)}=\left[\begin{array}{cc}
0 & 1 \\
-\left(2-\frac{\alpha}{\beta}-4 \frac{\alpha^{2}}{\beta^{2}}\right) & \xi-\frac{\alpha^{2}}{\beta^{2}} \delta
\end{array}\right] .
$$


The characteristic equation yields:

$$
\lambda^{2}-\left(\xi-\frac{\alpha^{2}}{\beta^{2}} \delta\right) \lambda+2-\frac{\alpha}{\beta}-4 \frac{\alpha^{2}}{\beta^{2}}=0 .
$$

Letting $p=-\left(\xi-\delta \alpha^{2} / \beta^{2}\right), q=2-\alpha / \beta-4 \alpha^{2} / \beta^{2}, \xi \ll 1$ and $\delta \ll 1$, the eigenvalues of the characteristic equation can be obtained as:

$$
\lambda_{1,2}=\frac{-p \pm i \sqrt{4 q-p^{2}}}{2} .
$$

It is worth pointing out, that the equilibria $\left(x_{4,5}, y_{4,5}\right)$ are stable focus for $p \leqslant 0$. On the other hand, the equilibria $\left(x_{4,5}, y_{4,5}\right)$ are unstable focus when $p>0$. It is found that system (3) has two semi-simple eigenvalues $\lambda_{1,2}= \pm \sqrt{q} i$ for $p=0$, the double Andronov-Hopf bifurcation occurs on $\xi / \delta=\alpha^{2} / \beta^{2}$. It is a necessary condition for Andronov-Hopf bifurcation.

\subsection{Homoclinic Bifurcation}

Here, we provide the details of Melnikov analysis for the system (3), expository discussions of theory can be found in [22, 23, 24]. Then, we introduce following notation in system (3):

$$
\begin{gathered}
F(X)=\left[\begin{array}{c}
y \\
\sin x(\alpha+\beta \cos x)
\end{array}\right], \quad G(X)=\left[\begin{array}{c}
0 \\
\left(\xi-\delta \cos ^{2} x\right) y
\end{array}\right], \quad X=\left[\begin{array}{l}
x \\
y
\end{array}\right], \\
\operatorname{Tr}(D F)=\operatorname{Tr}\left[\begin{array}{cc}
0 \\
\cos x(\alpha+\beta \cos x)-\beta \sin ^{2} x & 0
\end{array}\right]=0,
\end{gathered}
$$

and

$$
F(X) \wedge G(X)=\left(\xi-\delta \cos ^{2}\left(x_{ \pm}^{\text {hom }}(t)\right)\right)\left(y_{ \pm}^{\text {hom }}(t)\right)^{2},
$$

where $u \wedge v=u_{1} v_{2}-u_{2} v_{1}$ for any $u=\left(u_{1}, u_{2}\right)^{T}$ and $v=\left(v_{1}, v_{2}\right)^{T}$, the corresponding Melnikov function for the homoclinic orbits of the first and second type is given by:

$$
M^{h o m}(\xi, \delta)=\int_{-\infty}^{+\infty}\left(\xi-\delta \cos ^{2}\left(x_{ \pm}^{h o m}(t)\right)\right)\left(y_{ \pm}^{\text {hom }}(t)\right)^{2} d t
$$

Eq. (16) can be rewritten as

$$
M^{h o m 1,2}(\xi, \delta)=\xi I_{1}^{h o m 1,2}-\delta I_{2}^{h o m 1,2},
$$


where

$$
\begin{aligned}
& I_{1}^{h o m 1}=\int_{-\infty}^{+\infty}\left(y_{ \pm}^{h o m 1}(t)\right)^{2} d t \\
& =\int_{-\infty}^{+\infty}\left(\frac{\mp 2 \sqrt{-\alpha} \sinh (\sqrt{\alpha+\beta} t)}{1+\frac{-\alpha}{\alpha+\beta} \cosh ^{2}(\sqrt{\alpha+\beta} t)}\right)^{2} d t \\
& =4 \sqrt{\alpha+\beta}+4 \frac{\alpha}{\sqrt{\beta}} \ln \left(\sqrt{-\frac{\beta}{\alpha}}+\sqrt{-1-\frac{\beta}{\alpha}}\right), \\
& I_{2}^{\text {hom } 1}=\int_{-\infty}^{+\infty} \cos ^{2}\left(x_{ \pm}^{\text {hom } 1}(t)\right)\left(y_{ \pm}^{\text {hom } 1}(t)\right)^{2} d t \\
& =\int_{-\infty}^{+\infty} \cos ^{2}\left( \pm 2 \cot ^{-1}\left(\sqrt{\frac{-\alpha}{\alpha+\beta}} \cosh (\sqrt{\alpha+\beta} t)\right)\right) \\
& \times\left(\frac{\mp 2 \sqrt{-\alpha} \sinh (\sqrt{\alpha+\beta} t)}{1+\frac{-\alpha}{\alpha+\beta} \cosh ^{2}(\sqrt{\alpha+\beta} t)}\right)^{2} d t \\
& =\frac{A}{3(1+B)^{2.5}}\left(2\left(2+8 B+3 B^{2}\right) \sqrt{1+B}\right. \\
& \left.+3 B\left(2+2 B+B^{2}\right) \ln \left(\frac{2+B-2 \sqrt{1+B}}{B}\right)\right) \\
& A=\sqrt{\alpha+\beta}, \quad B=\frac{-\alpha}{\alpha+\beta} .
\end{aligned}
$$


and

$$
\begin{aligned}
I_{1}^{\text {hom } 2}= & \int_{-\infty}^{+\infty}\left(y_{ \pm}^{\text {hom } 2}(t)\right)^{2} d t \\
= & \int_{-\infty}^{+\infty}\left(\mp \frac{2 \sqrt{-\alpha} \cosh (\sqrt{\beta-\alpha} t)}{1+\frac{-\alpha}{\beta-\alpha} \sinh ^{2}(\sqrt{\beta-\alpha} t)} d t\right. \\
= & 4 \sqrt{\beta-\alpha}-4 \frac{\alpha}{\sqrt{\beta}} \ln \left(\sqrt{-\frac{\beta}{\alpha}}+\sqrt{1-\frac{\beta}{\alpha}}\right), \\
I_{2}^{\text {hom } 2}= & \int_{-\infty}^{+\infty} \cos ^{2}\left(x_{ \pm}^{\text {hom } 2}(t)\right)\left(y_{ \pm}^{\text {hom } 2}(t)\right)^{2} d t \\
= & \int_{-\infty}^{+\infty} \cos ^{2}\left( \pm 2 \tan ^{-1}\left(\sqrt{\frac{-\alpha}{\beta-\alpha}} \sinh (\sqrt{\beta-\alpha} t)\right)\right) \\
& \times\left(\frac{\mp 2 \sqrt{-\alpha} \cosh ^{2}(\sqrt{\beta-\alpha} t)}{1+\frac{-\alpha}{\beta-\alpha} \sinh ^{2}(\sqrt{\beta-\alpha} t)} d t\right. \\
= & \frac{C}{3(1-D)^{2.5}}\left(2\left(2-8 D+3 D^{2}\right) \sqrt{1-D}\right. \\
& \left.-3 D\left(2-2 D+D^{2}\right) \ln \left(\frac{2-D-2 \sqrt{1-D}}{D}\right)\right) \\
C & =\sqrt{\beta-\alpha}, \quad D=\frac{-\alpha}{\beta-\alpha} .
\end{aligned}
$$

It is worth pointing out, that the homoclinic bifurcations of the first and second type occur on $M^{h o m 1}(\xi, \delta)=0$ and $M^{h o m 2}(\xi, \delta)=0$, respectively.

\subsection{Closed Orbits Bifurcation}

In order to analyze multiple bifurcations and different types of limit cycles of system (3), we need to discuss the number of zero solutions of subharmonic Melnikov function. Let $(x(t), y(t))$ represent the closed periodic orbits inside or outside a pair of homoclinic loops $\Gamma_{h=0}$ and $\Gamma_{h=-2 \alpha}$. The Hamiltonian function is defined by Eq. (7), now the closed orbit $H(x(t), y(t))=h$ is satisfied with period $T$. Thus, the subharmonic Melnikov function can be obtained as:

$$
M(h)=\int_{0}^{T}\left(\xi-\delta \cos ^{2}(x(T))\right)(y(T))^{2} d t=\int_{H=h}\left(\xi-\delta \cos ^{2} x\right) y d x .
$$

Let $M(h)=0$, we have:

$$
P(h)=\frac{\xi}{\delta}=\frac{I_{2}(h)}{I_{1}(h)}
$$


where:

$$
\begin{aligned}
& I_{1}(h)=\int_{H=h} y d x=2 \int_{\boldsymbol{a}}^{\boldsymbol{b}} y(x) d x, \\
& I_{2}(h)=\int_{H=h} y \cos ^{2} x d x=2 \int_{\boldsymbol{a}}^{\boldsymbol{b}} y(x) \cos ^{2} x d x .
\end{aligned}
$$

The function $P(h)$ can be rewritten as:

$$
P(h)=\frac{\xi}{\delta}=\frac{\int_{\boldsymbol{a}}^{\boldsymbol{b}} y(x) \cos ^{2} x d x}{\int_{\boldsymbol{a}}^{\boldsymbol{b}} y(x) d x} .
$$

For a given closed orbit to remain after perturbation, we only require $M(h)=0$ or $P(h)=0$.

When $H(x(t), y(t))=h$, the Hamiltonian function (7) can be expressed $y$ as a function of $x$ :

$$
y(x)= \pm \sqrt{2 h-2 \alpha \cos x+\beta \sin ^{2} x+2 \alpha} .
$$

For $y(x)=0$, the trajectory of system (5) intersects the $x$-axis at $\left(\boldsymbol{a}_{i}, \boldsymbol{b}_{i}\right)_{i=1,2,3}$. Furthermore, the turning points $\left(\boldsymbol{a}_{i}, \boldsymbol{b}_{i}\right)_{i=1,2,3}$ can be obtained and classified in the following form:

$$
\begin{aligned}
& \text { (i) } h \in\left[-\frac{(\alpha+\beta)^{2}}{2 \beta}, 0\right) \text {, } \\
& \begin{array}{l}
\boldsymbol{a}_{1}=-\arccos \left(\frac{-\alpha-\sqrt{(\alpha+\beta)^{2}+2 \beta h}}{\beta}\right), \\
\boldsymbol{b}_{1}=\arccos \left(\frac{-\alpha+\sqrt{(\alpha+\beta)^{2}+2 \beta h}}{\beta}\right) ;
\end{array}
\end{aligned}
$$

(ii) $h \in[0,-2 \alpha)$,

$$
\begin{aligned}
& \boldsymbol{a}_{2}=-\arccos \left(\frac{-\alpha-\sqrt{(\alpha+\beta)^{2}+2 \beta h}}{\beta}\right), \\
& \boldsymbol{b}_{2}=\arccos \left(\frac{-\alpha-\sqrt{(\alpha+\beta)^{2}+2 \beta h}}{\beta}\right) ;
\end{aligned}
$$

(iii) $h \in[-2 \alpha,+\infty)$,

$$
\boldsymbol{a}_{3}=-\pi, \quad \boldsymbol{b}_{3}=\pi .
$$

Take $\alpha=-1$ and $\beta=2$ for example, the turning points $\left(\boldsymbol{a}_{i}, \boldsymbol{b}_{i}\right)_{i=1,2,3}$ are marked with solid circles in the Fig. 2(b).

\subsection{Multiple Bifurcations and Limit cycles}

Then, we can analyze the zeros of $P(h)$ and the associated level curves $P(h)=\zeta$ to investigate the number of limit cycles by using numerical integral 
method for $\alpha=-1$ and $\beta=2$. Therefore, the zeros of $M(h)$ can be determined by the intersection points of $P(h)$ and the level line $P(h)=\zeta$, as shown in Fig. $4\{h, P(h)\}$. Take $\alpha=-1$ and $\beta=2$ for example, more detailed classification is as follows:

(1) When $\zeta \in[0,0.25)$, there is no point of intersection between the curve $P(h)$ and the level curve $P(h)=\zeta$, which indicates that none closed orbit exists for $\zeta \in[0,0.25)$;

(2) For $\zeta \in\left[0.25, b_{1}\right)$, the curve $P(h)$ intersects the level curve $P(h)=\zeta$ at unique point for $h \in\left(-0.25, a_{1}\right)$. This implies, for $\zeta \in\left(0.25, b_{1}\right)$, there exist two closed orbits for $h \in\left(-0.25, a_{1}\right)$, encircling the singular points $\left(x_{4}, y_{4}\right)$ and $\left(x_{5}, y_{5}\right)$, respectively;

(3) Enlarging $\zeta$ to $b_{1}$ with $P^{\prime}\left(a_{6}\right)=0\left(P^{\prime}(h)=\partial P(h) / \partial h\right)$, the curve $P(h)$ and the level curve $P(h)=\zeta$ intersect at two points for $h=a_{1}, h=$ $a_{6}$. This suggests, for $\zeta=b_{1}$, there exist three closed orbits, two for $h=a_{1}$, encircling the singular points $\left(x_{4}, y_{4}\right)$ and $\left(x_{5}, y_{5}\right)$, respectively and another one for $h=a_{6}$ enclosing the singular points $\left(x_{1}, y_{1}\right)$ and $\left(x_{4,5}, y_{4,5}\right)$;

(4) When $\zeta \in\left(b_{1}, P(0)\right)$, the curve $P(h)$ meets the level curve $P(h)=\zeta$ at three points for $h \in\left(a_{1}, 0\right), h \in\left(a_{5}, a_{6}\right)$ and $h \in\left(a_{6}, a_{7}\right)$. There exist four closed orbits, two large closed orbits, encircling the singular points $\left(x_{1}, y_{1}\right)$ and $\left(x_{4,5}, y_{4,5}\right)$ for $h \in\left(a_{5}, a_{6}\right)$ and $h \in\left(a_{6}, a_{7}\right)$ and two small

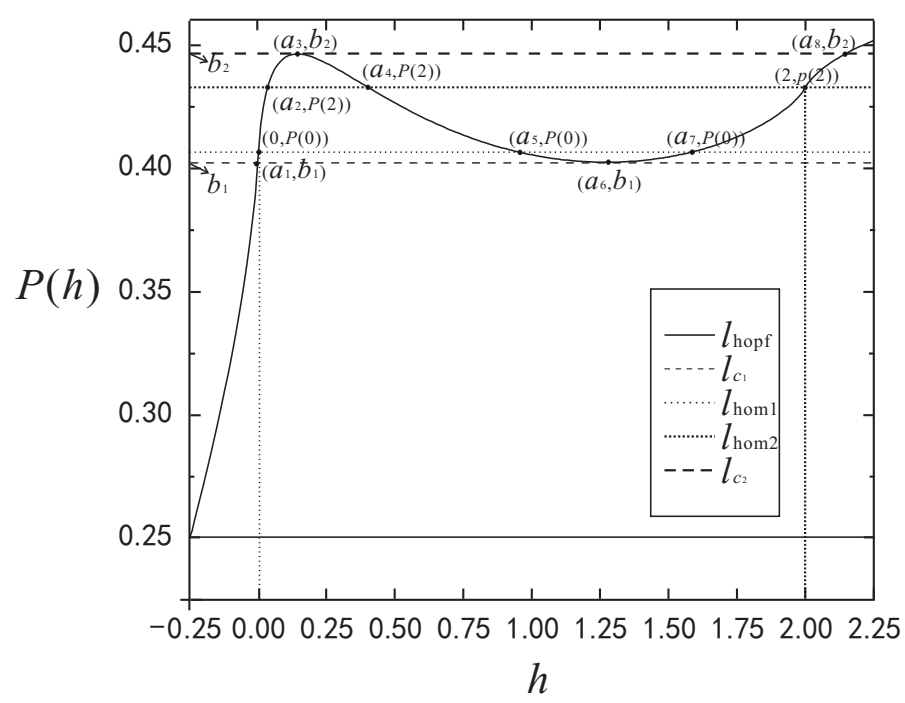

Fig. 4. The graph of curve $P(h)$ with associated preserved level curves $P(h)=\zeta$ 
closed orbits, encircling the singular points $\left(x_{4,5}, y_{4,5}\right)$ for $h \in\left(a_{1}, 0\right)$, respectively;

(5) For $\zeta=P(0), P(h)$ and $P(h)=\zeta$ intersect at three points for $h=0$, $h=a_{5}$ and $h=a_{7}$. This indicates, for $\zeta=P(0)$, there exist two large orbits, encircling the singular points $\left(x_{1}, y_{1}\right)$ and $\left(x_{4,5}, y_{4,5}\right)$ for $h=a_{5}$, $h=a_{7}$ and the homoclinic orbits of first type for $h=0$;

(6) Then $\zeta \in(P(0), P(2)), P(h)$ and $P(h)=\zeta$ meet at three points for $h \in\left(0, a_{2}\right), h \in\left(a_{4}, a_{5}\right)$ and $h \in\left(a_{7}, 2\right)$. This means, that there exist three large orbits, encircling the singular points $\left(x_{1}, y_{1}\right)$ and $\left(x_{4,5}, y_{4,5}\right)$;

(7) When $\zeta=P(2), P(h)$ intersects $P(h)=\zeta$ at three points for $h=a_{2}$, $h=a_{4}$ and $h=2$. This implies, for $\zeta=P(2)$, there exist two large closed orbits, encircling the singular points $\left(x_{1}, y_{1}\right)$ and $\left(x_{4,5}, y_{4,5}\right)$ and the homoclinic orbits of the second-type, connecting the singular points $\left(x_{2}, y_{2}\right)$ and $\left(x_{3}, y_{3}\right)$

(8) While $\zeta \in\left(P(2), b_{2}\right), P(h)$ and $P(h)=\zeta$ intersect at three points for $h \in\left(a_{2}, a_{3}\right), h \in\left(a_{3}, a_{4}\right)$ and $h \in\left(2, a_{8}\right)$. This means, for $\zeta \in\left(P(2), b_{2}\right)$, there exist two large rotational closed orbits and two large closed orbits, encircling the singular points $\left(x_{1}, y_{1}\right)$ and $\left(x_{4,5}, y_{4,5}\right)$;

(9) When $\zeta=b_{2}$ with $P^{\prime}\left(a_{3}\right)=0, P(h)$ intersects $P(h)=\zeta$ at two points for $h=a_{3}$ and $h=a_{8}$. It is found, that there exist two large rotational closed orbits and one large closed orbit, encircling the singular points $\left(x_{1}, y_{1}\right)$ and $\left(x_{4,5}, y_{4,5}\right)$

(10) For $\zeta \in\left(b_{2},+\infty\right), P(h)$ and $P(h)=\zeta$ intersect at one point for $h \in$ $\left(a_{8},+\infty\right)$. This implies, for $\zeta \in\left(b_{2},+\infty\right)$, there exist two large rotational closed orbits for $h \in\left(a_{8},+\infty\right)$.

Then, we define:

$$
\Sigma=l_{\text {hopf }} \cup l_{c_{1}} \cup l_{\text {hom } 1} \cup l_{\text {hom } 2} \cup l_{c_{2}},
$$

as the bifurcation diagrams for system (3) in parameter space $\{\xi, \delta\}$ plane. To illustrate the above theoretical analysis, the bifurcation diagram $\Sigma$ is plotted in Fig. $5(\Sigma)$ :

(1) The double Andronov-Hopf bifurcation $l_{\text {hopf }}$ with solid line:

$$
\delta-4 \xi=0
$$

(2) The first saddle-node bifurcation of periodic orbits bifurcation $l_{c_{1}}$ marked dashed line:

$$
\delta-c_{1} \xi=0, c_{1}=1 / b_{1} \approx 2.4956
$$




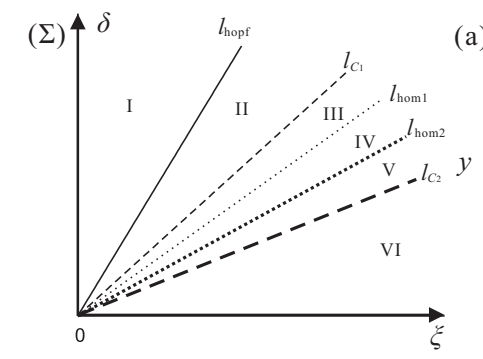

(c)
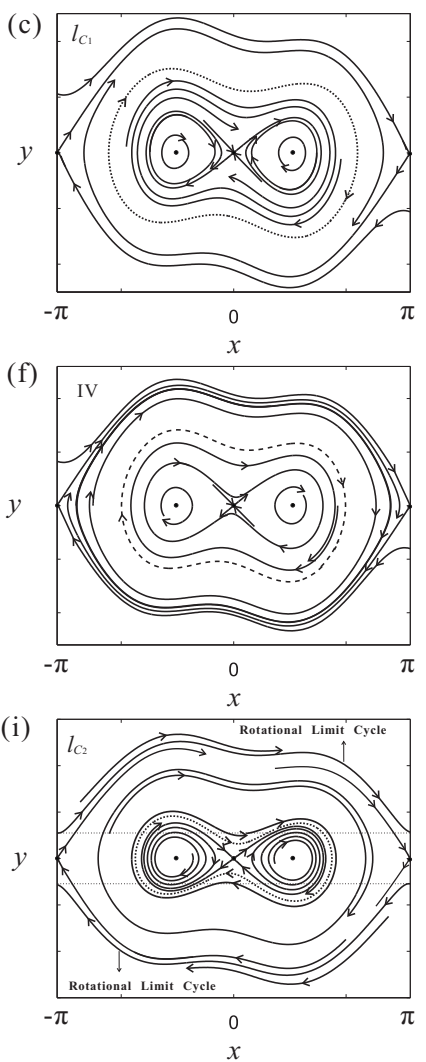

(a)

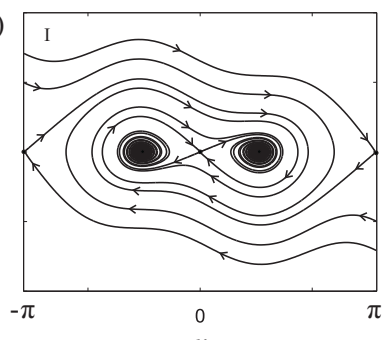

(d)

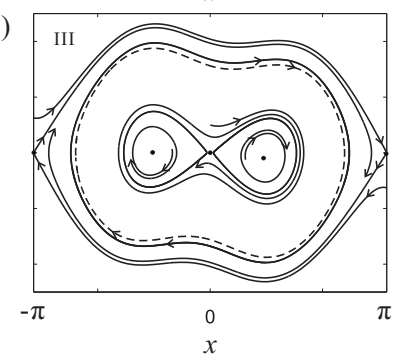

(g)

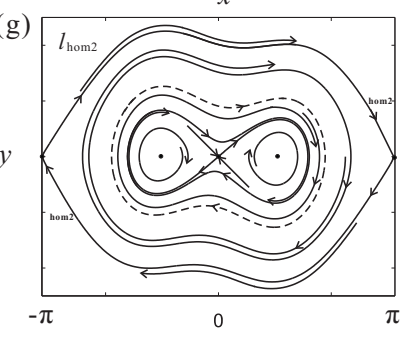

(j)

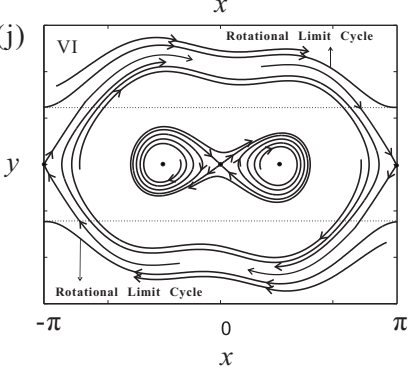

(b)

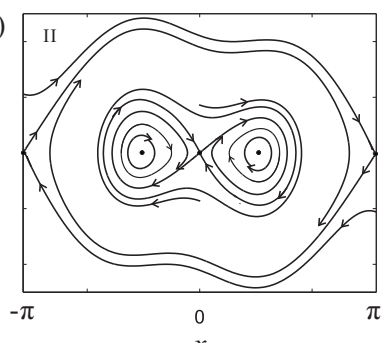

(e)

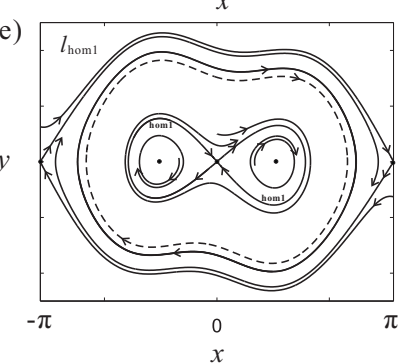

(h)

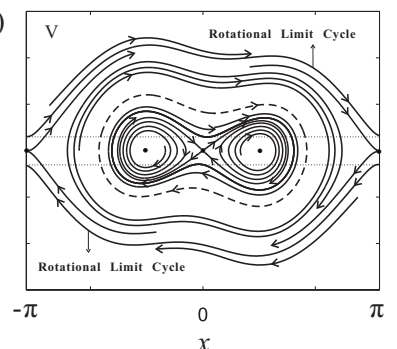

Fig. 5. Bifurcation diagrams $(\Sigma)$ and the corresponding perturbed dynamics (a)-(j)

(3) The homoclinic bifurcation of the first type $l_{h o m 1}$, marked dotted line:

$$
(\sqrt{2} \ln (3-2 \sqrt{2})+4) \xi-\left(\frac{5 \sqrt{2}}{8} \ln (3-2 \sqrt{2})+\frac{13}{6}\right) \delta=0
$$

(4) The homoclinic bifurcation of the second type $l_{h o m 2}$ with thick dotted 

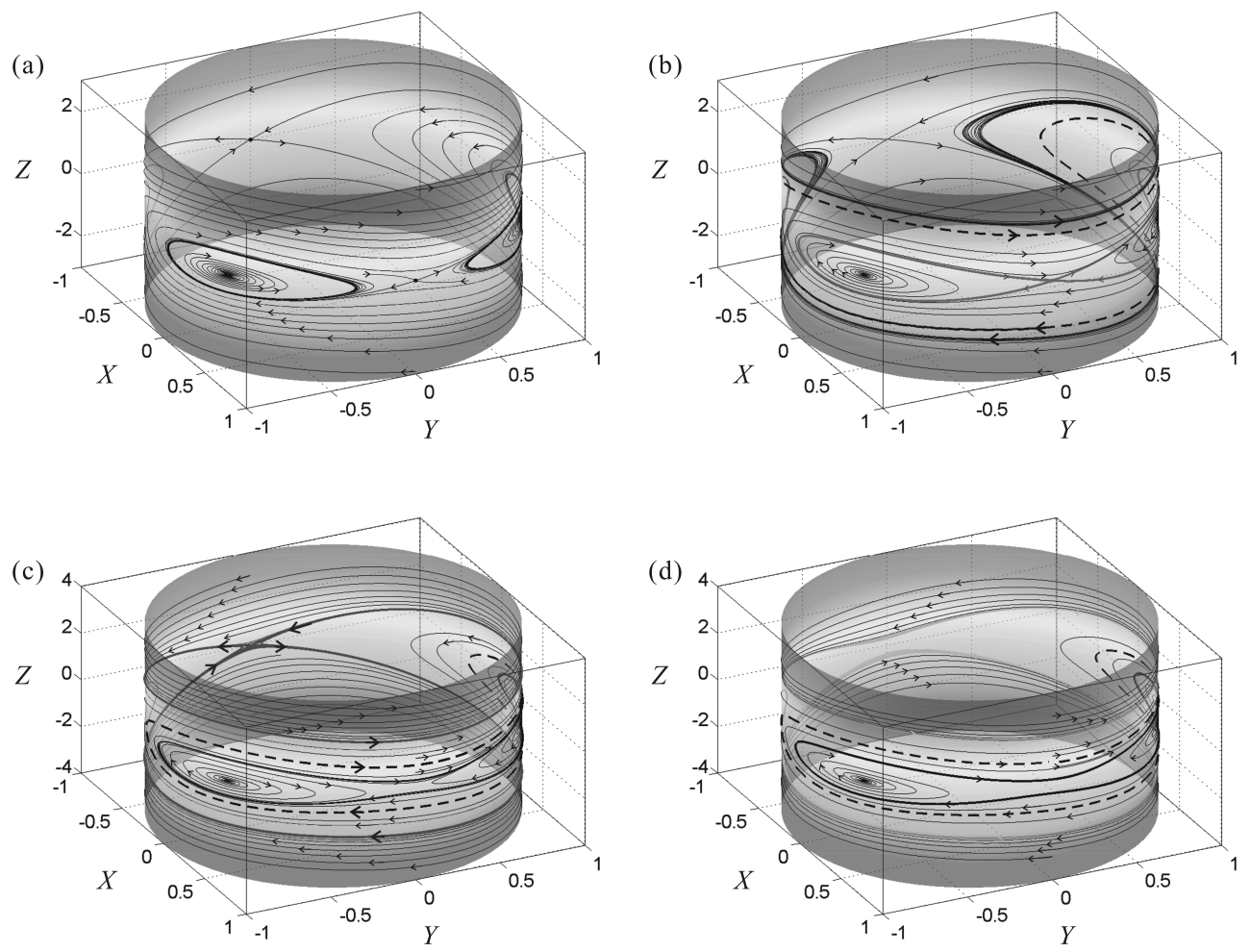

Fig. 6. (a). A pair of small oscillating limit cycles for double Andronov-Hopf bifurcation; (b). the homoclinic orbits of first type and two large oscillating limit cycles; (c). the homoclinic orbits of second type and two large oscillating limit cycles; (d). the coexistence of large oscillating limit cycles and rotational limit cycles

line,

$$
(\sqrt{2} \ln (5+2 \sqrt{6})+4 \sqrt{3}) \xi-\left(\frac{13 \sqrt{2}}{8} \ln (5+2 \sqrt{6})-\frac{\sqrt{3}}{2}\right) \delta=0 ;
$$

(5) The second saddle-node bifurcation of periodic orbits bifurcation $l_{c_{2}}$ denoted thick dashed line,

$$
\delta-c_{2} \xi=0, c_{2}=1 / b_{2} \approx 2.28 .
$$

It is worth pointing out, that the bifurcation curves divide the two dimensional parameter space $\{\xi, \delta\}$ into six persistence regions, denoted as I, II, III, IV, V and VI, respectively. Meanwhile, the stability, the number and position of the limit cycles in every region and bifurcation curves are displayed in Fig. 5(a)-(j).

When $(\xi, \delta)$ locates into region $\mathbf{I}$, three saddles $\left(x_{1,2,3}, y_{1,2,3}\right)$ and two 
stable focusses $\left(x_{4,5}, y_{4,5}\right)$ are displayed without limit cycle, as shown in Fig. 5(a). When $(\xi, \delta)$ touches the branch $l_{h o p f}$, there appears a double Andronov-Hopf bifurcation at the equilibria $\left(x_{4,5}, y_{4,5}\right)$ in Fig. $5(\mathrm{~b})$, which bifurcates into a pair of small oscillating limit cycles (stable), encircling the equilibria $\left(x_{4}, y_{4}\right)$ and $\left(x_{5}, y_{5}\right)$, respectively, when $(\xi, \delta)$ enters into region II.

While $(\xi, \delta)$ touches the branch $l_{c_{1}}$, there appears one saddle-node bifurcation of periodic solution in Fig. 5(c). It is found, that there exist semi-stable large oscillating limit cycles, encircling the equilibria $\left(x_{1}, y_{1}\right)$ and $\left(x_{4,5}, y_{4,5}\right)$, marked dotted line, coexisted with the pair of small oscillating limit cycles, encircling the equilibria $\left(x_{4}, y_{4}\right)$ and $\left(x_{5}, y_{5}\right)$, respectively.

As $(\xi, \delta)$ crosses the branch $l_{c_{1}}$ and locates into region III, the semistable limit cycles bifurcate into two large oscillating limit cycles, encircling the equilibria $\left(x_{1}, y_{1}\right)$ and $\left(x_{4,5}, y_{4,5}\right)$. One of them is stable denoted by solid line, the other is unstable marked by dashed line. Meanwhile there are a pair of stable small oscillating limit cycles, encircling the equilibria $\left(x_{4}, y_{4}\right)$ and $\left(x_{5}, y_{5}\right)$, respectively, as shown in Fig. 5(d).

A pair of stable small oscillating limit cycles encircling the equilibria $\left(x_{4}, y_{4}\right)$ and $\left(x_{5}, y_{5}\right)$ extend into a double-connected homoclinic orbits of the first type (hom 1 ), encircling the pair of unstable focusses in Fig. 5(e). A pair of large oscillating limit cycles encircling the equilibria $\left(x_{1}, y_{1}\right)$ and $\left(x_{4,5}, y_{4,5}\right)$ can be plotted outside of the homoclinic orbits of the first-type with solid line (stable) and dashed line (unstable).

When $(\xi, \delta)$ across $l_{\text {hom } 1}$ into region $\mathbf{I V}$, this double-connected homoclinic orbits of the first type bifurcates into a stable large oscillating limit cycle, encircling the pair of the unstable focuses and the saddle, embraced by a pair of limit cycles with solid line (stable) and dashed line (unstable), as shown in Fig. 5(f).

When $(\xi, \delta)$ crosses the branch $l_{h o m 2}$, the stable large limit cycle on the furthest exterior in Fig. 5(f) extend into a double-connected homoclinic orbits of the second type (hom 2$)$ connecting the saddle $\left(x_{2}, y_{2}\right)$ and $\left(x_{3}, y_{3}\right)$ in Fig. 5(g). It is noting, that there are a pair of oscillating limit cycles with solid line (stable) and dashed line (unstable) encircling the equilibria $\left(x_{1}, y_{1}\right)$ and $\left(x_{4,5}, y_{4,5}\right)$ inside the double-connected homoclinic orbits of the second type.

When $(\xi, \delta)$ crosses the branch $l_{h o m 2}$ and locates into the region $\mathbf{V}$, branch $l_{c 2}$ and VI, the double-connected homoclinic orbits of the second type bifurcate into a pair of rotational limit cycles above and below the homoclinic orbits, as shown in Fig. 5(h)-(j). It is clear, that a pair of oscillating limit cycles with solid line (stable) and dashed line (unstable) encircling the equilibria $\left(x_{1}, y_{1}\right)$ and $\left(x_{4,5}, y_{4,5}\right)$ in Fig. $5(\mathrm{~h})$ concentrate in a semi-stable oscillating limit 
cycles with dotted line in Fig. 5(i) and then disappear in Fig. 5(j).

In order to more deeply understand multiple bifurcations and different types of limit cycles, we introduce cylindrical phase portraits to clearly describe the double Andronov-Hopf bifurcation, the homoclinic bifurcations and the coexistence of oscillating and rotational limit cycles, by means of the cylindrical system (4), as shown in Fig. 6. Due to the double Andronov-Hopf bifurcation, a pair of stable limit cycles can be found near equilibria $(1 / 2, \sqrt{3} / 2,0)$ and $(1 / 2,-\sqrt{3} / 2,0)$ in Fig. 6(a). Figure 6 (b) shows the homoclinic bifurcation of first type, including a homoclinic orbits of first type connecting the equilibria $(1,0,0)$ and two limit cycles, encircling the equilibria $(1,0,0),(1 / 2, \pm \sqrt{3} / 2,0)$. Figure 6(c) displays the homoclinic bifurcation of second type, including a homoclinic orbits of second type connecting the equilibria $(-1,0,0)$ and two limit cycles encircling the equilibria $(1,0,0),(1 / 2, \pm \sqrt{3} / 2,0)$. It is most interesting that the homoclinic orbits of the second type can bifurcate into a pair of rotational limit cycles around the unit cylinder, coexisting with a stable limit cycles with thick solid line and a unstable limit cycles with thick dash line, as shown in Fig. 6(d).

Table 1 . The number of limit cycle and their stability in every region

\begin{tabular}{lcccccc}
\hline$(\xi, \delta)$ & I & II & III & IV & V & VI \\
\hline Limit Cycle 1 & 0 & 2 S & 2 S & 0 & 0 & 0 \\
Limit Cycle 2 & 0 & 0 & 2 SU & 3 SUS & 2 US & 0 \\
Limit Cycle 3 & 0 & 0 & 0 & 0 & 2 SS & 2 SS \\
\hline
\end{tabular}

Table 2. The number of limit cycle and their stability in bifurcation curves

\begin{tabular}{lccccc}
\hline$(\xi, \delta)$ & $l_{h o p f}$ & $l_{c_{1}}$ & $l_{\text {hom } 1}$ & $l_{\text {hom } 2}$ & $l_{c_{2}}$ \\
\hline Limit Cycle 1 & 0 & $2 \mathbf{S}$ & 0 & 0 & 0 \\
Limit Cycle 2 & 0 & $1 \overline{\mathbf{S}}$ & $2 \mathbf{S U}$ & $2 \mathbf{S U}$ & $1 \overline{\mathbf{S}}$ \\
Limit Cycle 3 & 0 & 0 & 0 & 0 & $2 \mathbf{S S}$ \\
Double Hom 1 & 0 & 0 & $1 \mathbf{S}$ & 0 & 0 \\
Double Hom 2 & 0 & 0 & 0 & $1 \mathbf{S}$ & 0 \\
\hline
\end{tabular}

The number and the stability of limit cycles in every region and bifurcation curve are listed in Tables 1 and 2, respectively. The limit cycle 1, limit cycle 2 and limit cycle 3 represent three kinds of limit cycles, small oscillating limit cycle, encircling one singular point, large oscillating limit cycle, encircling three singular points and rotational limit cycle, surrounding unit cylinder, respectively. The double hom 1 and double hom 2 are on behalf of the double 
homoclinic orbits of first and second type. Meanwhile, the stability of limit cycles could be divided into stable, unstable and semi-stable, denoted by $\mathbf{S}, \mathbf{U}$ and $\overline{\mathbf{S}}$ in Tables 1 and 2, respectively.

\section{Conclusions}

In this paper, we have studied multiple bifurcations of a tipically cylindrical dynamical system, whose unperturbed system bears the coupling dynamics property of the bistable state and the conventional pendulum with the homoclinic orbits of the first and second type. In the perturbed system, a double Andronov-Hopf bifurcation, a pair of homoclinic bifurcations and two saddle-node bifurcations of periodic orbits have been investigated by using the analytical analysis and numerical calculation. The number, position and stability of all the oscillating and rotational limit cycles are given as the perturbed parameters vary. It has been shown, that the homoclinic orbits of the second type can bifurcate into a pair of rotational limit cycles, coexisting with the oscillating limit cycle for a given set of parameters. Meanwhile, we have discovered that there are four limit cycles in this cylindrical dynamical system. The contribution of this work is to provide a typical example to reveal the complex dynamic bifurcation and different types of limit cycles of cylindrical dynamical system. All the results related to the perturbed system are obtained by the fourth order Runge-Kutta method, which ensures the accuracy of the computation.

\section{REFERENCES}

[1] Guckenheimer, J., P. Holmes. Nonlinear Oscillations, Dynamical Systems, and Bifurcations of Vector Fields, New York, Springer, 1983.

[2] Yagasaki, K. A. Simple Feedback Control System: Bifurcations of Periodic Orbits and Chaos. Nonlinear Dyn., 9 (1996), 391-417.

[3] Colonius, F., L. GrÜne. Dynamics, Bifurcations and Control, Berlin, SpringerVerlag, 2002.

[4] Kim, Y. H., S. H. Kım, Y. K. Kwak. Dynamic Analysis of a Nonholonomic TwoWheeled Inverted Pendulum Robot. J. Intell. Robot. Syst., 44 (2005), 25-46.

[5] Anh, N. D., H. Matsuhisa, L. D. Viet, M. Yasuda. Vibration Control of an Inverted Pendulum Type Strcture by Passive Mass-Spring-Pendulum Dynamics Vibration Absorber. J. Sound Vib., 307 (2007), 187-201.

[6] Sorokin, A., F. Arnold. Electrically Charged Small Soot Particles in the Exhaust of an Aircraft Gas-Turbine Engine Combustor: Comparison of Model and Experiment. Atmos. Environ., 38 (2004), No. 17, 2611-2618. 
[7] Kwek, K. H., J. B. LI. Chaotic Dynamics and Subharmonic Bifurcations in a Nonlinear System. Int. J. Non-linear Mech., 31 (1996), No. 3, 277-295.

[8] Xu, X., M. Wiercigroch. Approximate Analytical Solutions for Oscillatory and Rotational Motion of a Parametric Pendulum. Nonlinear Dyn., 47 (2007), 311420.

[9] Yabuno, H., M. Miura, N. Aoshima. Bifurcation in an Inverted Pendulum with Tilted High-Frequency Excitation: Analytical and Experimental Investigations on the Symmetry Breaking of the Bifurcation. J. Sound Vib., 273 (2004), 493-513.

[10] Koch, B. P., R. W. Leven. Subharmonic and Homoclinic Bifurcations in a Parametrically Forced Pendulum. Physica D, 16 (1984), 1-13.

[11] CAO, Q. J., N. Han, R. L. Tian. A Rotating Pendulum Linked by an Oblique Spring. Chin. Phys. Lett., 28 (2011), No. 6, 060502:1-4.

[12] Cao, Q. J., M. Wiercigroch, E. E. Pavlovskaia, C. Grebogi, J. M. T. Thompson. An Archetypal Oscillator for Smooth and Discontinuous Dynamics. Phys. Rev. E, 74 (2006), 046218.

[13] Cao, Q. J., M. Wiercigroch, E. E. Pavlovskaia, C. Grebogi, J. M. T. Thompson. Piecewise Linear Approach to an Archetypal Oscillator for Smooth and Discontinuous Dynamics. Philos. Trans. R. Soc. A, 366 (2008), 635-652.

[14] CaO, Q. J., D. Wang, Y. S. Chen, M. Wiercigroch. Irrational Elliptic Functions and the Annalytical Solutions of SD Oscillator. J. Theor. Appl. Mech., 50 (2012), No. 3, 701-715.

[15] Han, N., Q. J. CaO. Estimation of the Chaotic Thresholds for the Recently Proposed Rotating Penulum. Int. J. Bifurcat. Chaos, 23 (2013), No. 4, 1350074: $1-22$.

[16] Han, N., Q. J. CaO. A Rotating Disk Linked by a Pair of Springs. Nonlinear Dyn., 79 (2015), 1275-1291.

[17] Chen, Y. S., J. Xu. Global Bifurcations and Chaos in a Van der Pol-Duffing Mathieu System with Three Well Potential Oscillator. Acta. Mechanica Sinica, 11 (1995), 354-372.

[18] Tang, J. S., J. Q. QIn, H. XiAo. Bifurcations of a Generalized Van der Pol Oscillator with Strong Nonlinearity. J. Sound Vib., 306 (2007), 890-896.

[19] Simpson, D. J. W., J. D. Meiss. Andronov-Hopf Bifurcations in Planar, Piecewise-Smooth, Continuous Flows. Phys. Lett. A, 371 (2007), 213-220.

[20] Tian, R. L., Q. J. CaO, S. P. Yang. The Codimension Two Bifurcation for the Recent Proposed SD-Oscillator. Nonlinear Dyn., 59 (2010), 19-27.

[21] Han, M. A. Global Behavior of Limit Cycles in Rotated Vector Fields. J. Differ. Equ., 151 (1999), 20-35.

[22] Melnikov, V. K. On the Stability of the Center for Time-Periodic Perturbations. Trans. Moscow Math., 12 (1963), 1-57.

[23] Wiggins, S. Global Bifurcations and Chaos, Analytical Methods, New York, Springer, 1988.

[24] Yagasaki, K. Chaos in a Pendulum with Feedback Control. Nonlinear Dyn., 6 (1994), 125-142. 